\title{
Correction to: The CsGPA1-CSAQPs module is essential for salt tolerance of cucumber seedlings
}

\author{
Yan Yan ${ }^{1} \cdot$ Mintao Sun ${ }^{1} \cdot$ Yansu $\mathrm{Li}^{1}$ · Jun Wang ${ }^{1} \cdot$ Chaoxing $\mathrm{He}^{1} \cdot$ Xianchang $\mathrm{Yu}^{1}$ (D) \\ Published online: 18 September 2021 \\ ○) Springer-Verlag GmbH Germany, part of Springer Nature 2021
}

\section{Correction to: Plant Cell Reports (2020) 39:1301-1316}

https://doi.org/10.1007/s00299-020-02565-5

Figure 5 in the original publication contains a mistake. It shows the interaction between CsGPA1 and CsCOR413PM2 instead of CsTIP1.1. The correct Fig. 5, showing the interaction between CsGPA1 and CsTIP1.1, is shown below.

The original article can be found online at https://doi.org/10.1007/ s00299-020-02565-5.

Chaoxing He

hechaoxing@caas.cn

$\triangle$ Xianchang Yu

yuxianchang@caas.cn

Yan Yan

yanyan@caas.cn

Mintao Sun

sunmintao@caas.cn

Yansu Li

liyansu@caas.cn

Jun Wang

wangjun01@ caas.cn

1 The Institute of Vegetables and Flowers, Chinese Academy of Agricultural Sciences, Haidian District, Zhongguancun South St, Beijing 100081, China 


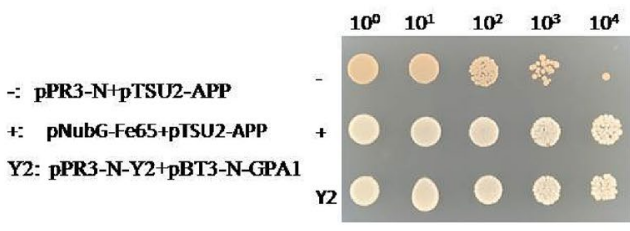

SD/-Leu/Trp

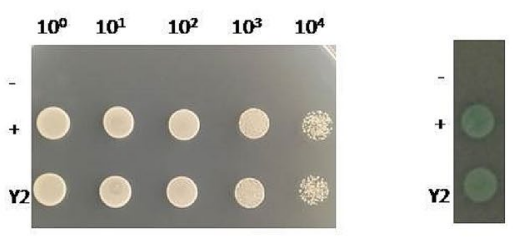

SD/Leu/-Trp/-His/-Ade $\quad$ SD/Leu/-Trp/-His/-Ade/X-gal

Fig. 5 Interaction between CsGPA1 and CsTIP1-1 using split-ubiquitin yeast two-hybrid System. a In SD/-Leu/-Trp, normal yeast colonies were grown proved that the vectors were successfully transferred into NMY51 yeast; b The negative control (-) did not produce colonies, indicating that there was no self-activation; c Color reaction.

Publisher's Note Springer Nature remains neutral with regard to jurisdictional claims in published maps and institutional affiliations.

'-' negative control, ' +' positive control, $Y 2$ yeast two-hybrid of the target genes, SD/-Leu/-Trp two deficient media, SD/-Trp-Leu-His-Ade four deficient media, $X$-gal $\beta$-galactosidase, $10^{n}$ is the dilution factor of yeast $(n=0,1,2,3,4)$ 